\title{
Developing a Theory-Informed Interactive Animation to Increase Physical Activity among Young People with Asthma
}

\author{
Jennifer Murray ${ }^{1}$, Brian Williams ${ }^{2}$, Gaylor Hoskins ${ }^{2}$, John McGhee ${ }^{3}$, Dylan Gauld ${ }^{3}$, \\ and Gordon Brown ${ }^{4}$ \\ ${ }^{1}$ Edinburgh Napier University, School of Life, Sport and Social Sciences, Edinburgh, Scotland \\ j.murray2@napier.ac.uk \\ ${ }^{2}$ Nursing, Midwifery and Allied Health Professions Research Unit, University of Stirling, \\ Stirling, Scotland \\ \{brian.williams, Gaylor.hoskins\} astir.ac.uk \\ ${ }^{3}$ Duncan of Jordanstone College of Art and Design, University of Dundee, Dundee, Scotland \\ $\{j . m c g h e e, d . g a u l d\}$ @dundee.ac.uk \\ ${ }^{4}$ Asthma UK Scotland, Edinburgh, Scotland \\ gbrowneasthma.co.uk
}

\begin{abstract}
The current paper describes the development of a theory-informed interactive animation and which aims to increase levels of physical activity in young people with asthma. The project adopts a multi-disciplinary theoretical perspective, applying knowledge from applied health research, human centred design and psychology in order to best approach and develop a meaningful and effective health intervention.
\end{abstract}

Keywords: Asthma, interactive animation, multidisciplinary, theory-informed.

\section{Introduction}

To begin, an introduction to the issues surrounding physical activity and young people with asthma will be discussed, to provide a context for the need for the development of the theory-informed interactive animation. The key theoretical bases informing the development of the animation are then discussed, as are the key HCI principles that apply to the animation's development. This is then followed by a broad description of initial assessments of the animation and future plans to assess and refine it.

\section{Background}

Within the UK, the number of new diagnoses of asthma has increased significantly over the past 20 years [1]; one in five children is now affected by asthma within the UK [2,3]. One in five of all child GP consultations relate to asthma [4], and within England and Wales in 1999 alone there were over 30,000 asthma related hospital 
admissions and 25 related child deaths [5]. Improving asthma control is a necessity for both the health of young people and for reducing the burden on health services. One simple and cost effective way to do this is through physical activity; which can lead to improvements in aerobic fitness [6,7] and asthma related benefits such as reduced hospital admissions, reduced absences at school, reduced medication use [8] and improved ability to cope with asthma [7]. However, young people with asthma are actually less likely to be physically active than their peers [9-13], and that they attribute this to their asthma. For example, in a survey of young people in Los Angeles between 1999-2000, 53\% of those with a diagnosis of asthma reported their activities were limited because of their asthma [14]. The overwhelming majority of studies, however, indicate that young people with asthma can exercise safely when appropriately treated [15].

\section{Theoretical Basis}

We drew on the research team's extensive background in asthma and in developing innovative theoretically-informed visualisations to encourage behaviour change to develop the interactive animation. In depth qualitative work in the area of asthma and physical activity carried out previously by members [16] of the research team identified important beliefs that act as the principle reasons for low activity among young people with asthma. The study also found that young people were unable to distinguish when their asthma was the cause of shortness of breath, or when they were simply short of breath due to normal physical processes, and this then impacted on concerns for their safety and beliefs about their ability to take part in physical activity [16]. Subsequently, motivation to be physically active was diminished, and issues around self-efficacy (i.e., the level of belief that a person has about their capabilities) arose.

Thus the animation that we have developed aims to address these issues. First, by using the psychological literature on motivation, self-efficacy and learning theory (how young people learn most effectively) it is believed that the optimal learning environment can be created to enhance the user's experience of the animation and the messages given, encouraging them to take control (thus increasing self efficacy) and increasing motivation to alter behaviours and beliefs. There is insufficient scope within the context of the current paper to discuss these important elements in greater detail; however the authors plan to publish this information in future articles.

Second, previous work by the team in developing novel theory-informed animations has shown some success in changing health related behaviours through transforming 'abstract' concepts into 'concrete' ones. For example, an animation was developed using behavioural theory to create a 3D animation to illustrate the link between obesity and atherosclerosis, showing the user what was actually happening to the heart and blood vessels when weight was increased and decreased. We therefore 
applied this concrete-to-abstract method to the current project's animation, demonstrating what is happening inside the lungs during physical activity [17]. Williams and colleagues [17] found that through showing patients an animation detailing the impact of weight on the health of blood vessels and the heart, patients were able to better conceptualise what was happening within their own bodies, leading to increased motivation to alter behaviour and increased self-efficacy (i.e., the belief that they can change their behaviour).

We build on this research using the same key principle of transforming the 'abstract' concept of visualising the internal effects on the lungs and bronchioles during breathlessness due to asthma compared to normal breathlessness to a 'concrete' visualisation within the animation. This progression from an abstract concept to a concrete representation is thought to not only aid understanding and conceptualisation $[17,18]$, but also aid retention in memory [19]. Thus, through strengthening the concreteness of the visualisation, young people with asthma may be more likely to: 1) recall the lessons given within the animation; 2) improve their conceptualisation, thus understanding of the issue and; 3) through their increased understanding, begin to develop a better awareness of when they are breathless due to asthma versus when they are breathless simply due to normal physical exertion.

A key difficulty in current health based interventions which aim to alter behaviours lies in the translation of intentions to become more physically active to actually becoming more physically active: it is well known within the psychological literature that intentions do not always guarantee behaviour change. Put simply, the mere statement of intention to alter a behaviour does not always mean that the behaviour is altered in real life. We identified two key theories of behaviour change which were highly relevant to our topic area and applied these while designing our animation: the Theory of Planned Behaviour [20] and Leventhal's Common Sense Model of behaviour [21].

These models aim to explain why people behave in certain ways and what barriers and facilitators exist between the intention to act and the actual behaviour. Once again, the full discussion of these theories in relation to the development of the current animation is outwith the scope of the current paper, but will be discussed in future papers.

Addressing these psychological elements and applying theoretical models within our animation was therefore of paramount importance in developing a useful, evidence based intervention.

\section{Developing and Assessing the Animation}

Using the theoretical bases described above, we have shaped the animation in such a way as to maintain a strong narrative, interactive elements to enhance engagement and immersion, and the animation that we have developed allows the user to select a character (choice of six, with initial testing piloting a choice of two, who will acts as a 'buddy') and a type of activity for the character to participate in (running, soccer or dance, with initial testing only piloting running). The characters introduce themselves 
to the user and discuss in a 'chatty', friendly tone their asthma and their inhaler use. The user is prompted to, at set points, decide whether their character will use inhalers or not; and they then see the consequences of this action. Both external views of the characters and internal views of the lungs are shown, to aid the user in establishing a concrete conceptualisation of what happens within the lungs during breathlessness caused by asthma compared to normal breathlessness, and what happens when inhalers are used correctly.

Based on lessons learned previously, we sought not only to embed psychological and health theory to the current animation, but to also embed human centred design principles to a greater degree and involve an online consultative user-group feedback mechanism throughout the design process at each key decision making stage.

The consultative user-group consisted of a representative sample 23 people, including young people with asthma $(\mathrm{N}=5)$, parents $(\mathrm{N}=4)$, teachers $(\mathrm{N}=6)$, adults with asthma $(\mathrm{N}=3)$ and health professionals $(\mathrm{N}=5)$ who met online to discuss general issues surrounding asthma and physical activities and more project-specific aspects (e.g., design preferences). General discussions with the user-group were found to be very fruitful in shaping our initial ideas and thoughts on the project; more so than expected. These discussions began with a prompt question or discussion point (e.g., which forms of physical activity do you enjoy most and least' and 'are there any types of physical activity that you feel your asthma stops you doing?'), and the users would discuss this in a typical online forum format, with members of the research team contributing additional prompts for follow-up information. These types of questions allowed us to form rapport with our user-group prior to asking for feedback on specific elements of the animation and allowed bonding between the members of the user group to allow an open and comfortable feedback and discussion platform for the project. It also allowed us to identify whether there were common themes in terms of likes, dislikes and general attitudes, helping us to form initial storyboards and concepts for the animation.

More specific feedback was sought from the user group through the use of closed or short-text surveys on specific components of the animation. In some cases these surveys were expanded and advertised on asthma-related forums and social networking pages to maximise feedback. This form of feedback informed development was particularly useful when designing the characters to be used within the animation. The user is able to select one of six characters. At the initial concept stage we canvassed opinions on likability, realism and general attitudes towards the characters. All but one character had some alterations made as a direct result of the feedback and two were substantially revised due to lack of popularity on multiple character-elements by the sample.

This was of particular importance, as one of the key design principles that we sought to embed within the animation was that of empathising with the characters. Through creating an empathic bond with the character, users are more likely to pay more attention and become engaged in [22] and immersed in [23] the animation's narrative. Having an empathic bond with the character and increased engagement and immersion within the animation should, therefore, lead to optimal learning and conceptualisation of the target information (i.e., the impact of physical activity and 
inhaler use on the lungs) and encourage users to utilise this in their future understandings and decisions about their physical activity in relation to their asthma.

The final key design principles adhered to included: the use of visual and audio narratives to enhance immersion and engagement [24]; the use of interactivity to allow choice, user engagement, and immersion [23]; allowing customisation of characters and physical activity scenarios to enhance user engagement and immersion [23]; the use of minimal additional text to enhance learning and comprehension but not detract from the visual narrative; and ensuring cognitive load was not too high when multiple messages were given to enhance comprehension.

As previously discussed, the 'look' of the animation was shaped by user-group feedback. We plan to carry out a more detailed and rigorous analysis of the effectiveness of the animation and its acceptability to key stakeholders (young people with asthma, parents, teachers, health professionals) via in-depth qualitative interviews and through an online interactive modelling experiment.

\section{Conclusion}

We hypothesise that through this rigorous blend of multidisciplinary theory and through the use of high quality design, the final animation will be highly attractive for use by health professionals as an intervention and will appeal to young people, their parents and to other key stakeholders. Through developing health (and potentially other) interventions in this way, it is hypothesised that behaviour and attitudinal change can be achieved to the benefit of target users.

\section{References}

1. Anandan, C., Nurmatov, U., Schayck, O., Sheikh, A.: Is the prevalence of asthma declining? Systematic review of epidemiological studies. Allergy Asthma Proc. (2009), doi:10.1111/j.1398-9995.2009.02244.x

2. Asher, M.I., Montefort, S., Bjorksten, B., Lai, C.K.W., Strachan, D.P., Weiland, S.K., et al.: Worldwide time trends in the prevalence of symptoms of asthma, allergic rhinoconjunctivitis, and eczema in childhood: ISAAC Phases One and Three repeat multicountry cross-sectional surveys. The Lancet 368(9537), 733-743 (2006)

3. Beasley, R.: The International Study of Asthma and Allergies in Childhood (ISAAC) Steering Committee. Worldwide variation in prevalence of symptoms of asthma, allergic rhinoconjunctivitis, and atopic eczema: ISAAC. The Lancet 351(9111), 1225-1232 (1998)

4. National Asthma Campaign. An audit of children's asthma in the UK. The Asthma Journal 8(2), 3-11 (2002)

5. National Asthma Campaign. Out in the open. A true picture of asthma in the United Kingdom today. The Asthma Journal 6(3), 3-14 (2001)

6. Ram, F.S.F., Robinson, S.M., Black, P.N.: Effects of physical training in asthma: a systematic review. Br. J. Sports Med. 34, 162-167 (2000)

7. van Veldhoven, N.H.M.J., Vermeer, A., Bogaard, J.M., Hessels, M.G.P., Wijnroks, L., Colland, V.T., et al.: Children with asthma and physical exercise: effects of an exercise programme. Clin. Rehabil. 15, 360-370 (2001) 
8. Welsh, L., Kemp, J.G., Roberts, R.G.D.: Effects of physical conditioning on children and adolescents with asthma. Sports Med. 35(2), 127-141 (2005)

9. Glazebrook, C., McPherson, A.C., Macdonald, I.A., Swift, J.A., Ramsay, C., Newbould, R., Smyth, A.: Asthma as a barrier to children's physical activity: implications for body mass index and mental health. Pediatrics 118(6), 2443-2449 (2006)

10. Lang, D.M., Butz, A.M., Duggan, A.K., Serwint, J.R.: Physical activity in urban school-aged children with asthma. Pediatrics 113, 341-346 (2004)

11. Meyer, A., Machnick, M.A., Behnke, W., Braumann, K.M.: Participation of asthmaic children in gymnastic lessons at school. Pneumologie 56(8), 486-492 (2002)

12. Sawyer, S.M., Fardy, H.J.: Bridging the gap between doctors' and patients' expectations of asthma management. J. Asthma 40(2), 131-138 (2003)

13. Yeatts, K., Sotir, M., Music, S., Herget, C.: Health consequences for children with undiagnosed asthma-like symptoms. Arch. Pediatr. Adolesc. Med. 157, 540-544 (2003)

14. Los Angeles County Department of Health Services. Los Angeles County health survey. Los Angeles: County of Los Angeles Department of Health Services Public Health (2001), http: //publichealth. lacounty.gov/ha/reports/habriefs/v3i6_as thma/asthm.pdf

15. Lucas, S.R., Platts-Mills, T.A.E.: Physical activity and exercise in asthma: relevance to etiology and treatment. J. Allergy Clin. Immunol. 115(5), 928-934 (2005)

16. Williams, B., Hoskins, G., Pow, J., Mukhoadhyay, S., Coyle, J.: Low exercise among children with asthma: A culture of over protection? A qualitative study of experiences and beliefs. British Journal of General Practice 60(577), e319-e326 (2010)

17. Williams, B., Anderson, A., Barton, K., McGhee, J.: Can theory be embedded in visual interventions to promote self-management? A proposed model and worked example. International Journal of Nursing Studies 49(12), 1598-1609 (2012)

18. Rich, M., Chalfen, R.: Showing and telling asthma: Children teaching physicians with visual narrative. Visual Sociology 14(1), 51-71 (1999)

19. Walker, I., Hulme, C.: Concrete words are easier to recall than abstract words: Evidence for a semantic contribution to short-term serial recall. Journal of Experimental Psychology: Learning, Memory, and Cognition 25(5), 1256-1271 (1999)

20. Ajzen, I.: The theory of planned behaviour. Organisational Behavior and Decision Processes 50, 179-211 (1991)

21. Leventhal, H., Brisette, I., Leventhal, E.A.: The common-sense model of self-regulation of health and illness. In: Cameron, L.D., Leventhal, H. (eds.) The Self Regulation of Health and Illness Behaviour, pp. 42-65. Routelidge, London (2003)

22. Kennedy, D.M., McNaught, C.: Design elements for interactive multimedia. Australian Journal of Educational Technology 13(1), 1-22 (1997)

23. Keen, S.: Fast tracks to narrative empathy: Anthropomorphism and dehumanisation in graphic narratives. SubStance 40(124), 135-155 (2011)

24. Plowman, L.: Narrative, linearity and interactivity: making sense of interactive multimedia. British Journal of Educational Technology 27(2), 92-105 (1996) 